\title{
Axonal growth and pathfinding: from phenomena to molecules
}

\author{
U. Drescher ${ }^{1}$, A. Faissner ${ }^{2}$, R. Klein ${ }^{3}$, F.G. Rathjen ${ }^{4}$, C. Stürmer ${ }^{5}$ \\ ${ }^{1}$ Max-Planck Institute for Developmental Biology, D-72076 Tübingen, Germany \\ 2 Department of Neurobiology, University of Heidelberg, Heidelberg, Germany \\ ${ }^{3}$ European Molecular Biology Laboratories, Heidelberg, Germany \\ ${ }^{4}$ Max-Delbrück Center for Molecular Medicine, Berlin, Germany \\ ${ }^{5}$ Neurobiology, University of Konstanz, Konstanz, Germany
}

Received: 10 July 1997

A long-standing goal of developmental neurobiology is to characterize the molecular mechanisms underlying the formation of functional connections during the development of the nervous system. These connections are primarily established by the elongation of axons along restricted pathways. A specialized tip of an extending axon, the so-called growth cone, continuously explores the local environment for guidance information and makes choices in certain places. The growth cone displays a peripheral motile region from which filopodia protrude. These filopodia can extend tens of microns from the central region of the growth cone and are therefore able to survey a large area in a relatively short period. Filopodia are thus important sensors of the growth cone. The detection of local guidance cues depends on the expression of receptor complexes that lie on the surface of the extending growth cone and that probably transduce extracellular signals into intracellular events to direct the growth cone. Cell biological studies have shown that the molecular signals that might affect the behavior of growth cones include diffusible and substrate-bound factors that act as attractants or repellents.

Research over the past decade has focused on the identification of molecular components expressed on the surface of extending axons and found in the environment of axons. These investigations have led to the identification of a variety of components that influence extending axons; these components are reviewed in this Special Issue of Cell and Tissue Research.

A relatively recently discovered family of short-range repulsive guidance cues is represented by the family of Eph receptors and their cell-surface ligands. Although the ligands have only been discovered during the last three years, remarkable progress has been made in elucidating the functions of these molecules. The Eph family represents by far the largest family of receptor tyrosine kinases, comprising at present 14 members. Eight ligands, termed ephrins, can be subdivided into two classes, based on the mode of membrane anchorage, viz., Ephrin A (gly-

Correspondence to: U. Drescher cosyl-phosphatidyl-inositol-anchored) and Ephrin B (transmembrane ligands). Because of their binding specificities, the Eph receptors can also be subdivided into two classes, EphA (interacting with A-Ephrins) and EphB (interacting with B-Ephrins). Some of the special features of these families include: (1) considerable promiscuity in binding within the EphA and EphB subclasses, in that nearly every ligand of a certain subclass can interact with almost all receptors of the corresponding subclass; (2) ligands can activate their receptors only in membranebound form; (3) when stimulated, these receptors do not evoke a mitogenic response, which is normally observed for the superfamily of receptor tyrosine kinases; and (4) in certain situations, the Ephrins can function both as a receptor and as a ligand. The Eph receptors can also have a pure ligand function in that the kinase domain is dispensable.

In vitro assays have implicated ephrins in the guidance of retinotectal projections and axon fasciculation. Expression gradients in various parts of the brain suggest their involvement in the establishment of many topographic projections. Gene knockout experiments have provided strong evidence that certain Eph receptors are required for the development of commissural axon projections. Moreover, the simultaneous elimination of two genes has shown that Eph receptors cooperate in axonal guidance, presumably because of their overlapping expression patterns and rather promiscuous binding activities. More recently, ephrins and their receptors have also been shown to guide migrating neural crest cells, suggesting that the signal transduction pathways that regulate axonal guidance and cell migration share many features. In view of the large number of Eph receptors and ligands, these molecules may be among the major determinants of the guidance of axons and cells in the developing nervous system.

It is well established that interactions between neurons and astrocytes are of importance in regulating the establishment of interneuronal connections. In addition to this role during development, astrocytes are also thought to play a role in the context of the inhibition of axonal regeneration in situations where these cells form a glial 
scar. Thus, astrocytes may exert ambivalent influences on fiber outgrowth, being stimulatory in some cases and providing transient or stable tissue boundaries in others. Various gene families are potentially involved in mediating these influences, and both stimulatory and inhibitory components have been described. Ig-superfamily members and cadherins are thought to underlie calcium-dependent and calcium-independent adhesive stimulatory interactions, respectively. Members of the Ig-superfamily appear to be one of the largest groups of proteins expressed on extending axons. These proteins have been primarily identified by immunological and genetic strategies and have been implicated in various aspects of neurohistogenesis, including contact-dependent axon guidance. Several of the Ig-family members are expressed in an overlapping manner on several axon systems during development, suggesting functional cooperation. Recent studies consistently indicate that neural Ig-superfamily members undergo complex interactions to regulate axon extension. The multivalency is in agreement with their modular domain organization.

In addition to Eph receptors and their ligands, components such as the semaphorins are strong candidates for exerting inhibitory influences in developing neural tissues. The semaphorins have been described in several species ranging from Drosophila to humans and are characterized by a conserved domain. Interestingly, some of the semaphorins are strongly associated with cell membranes and hence positionally fixed, whereas others are released into the pericellular environment and could diffuse for some distance through tissues. Thus, semaphorins (at least some of them) might build up gradients in the pericellular environment.

This is also true for components of the extracellularmatrix (ECM). The ECM can be subdivided into two biochemically distinct classes, viz., glycoproteins and proteoglycans, the latter consisting of a glycoprotein core and at least one additional covalently bound glycosaminoglycan side chain. Both classes of components are expressed in the central nervous system, and increasing evidence suggests that ECM constituents exert definable influences on axon growth and guidance. Promoting influ- ences have been described, e.g., for the laminins and the laminin-related netrin gene products, which are believed to build up attractive gradients for certain subclasses of axons during the development of the spinal cord. On the other hand, also repulsive influences have been described for the netrins acting on other classes of neurons. Similarly, the tenascins deflect most types of axons in choice situations where the glycoproteins are offered as a step gradient to advancing growth cones. Analogous observations have been reported for proteoglycans, although most of the chondroitin-sulfate-type proteoglycans described so far are thought to exert inhibitory effects on axon growth. These observations suggest that the pericellular space, which changes considerably during development, is structured by a variety of ECM components that form an organized, topologically specified, interactive superstructure with distinct effects on neuron precursor migration and growth cone advance. The available evidence supports the notion that the ECM components are deposited in controlled ways, e.g., as gradients in some cases or as boundaries in others. Thus, the ECM also seems to be part of the more complex control and signaling system that underlies the exquisite pathway choices of evolving fiber systems during development.

The relative contribution of these distinct gene families compared with others discussed in the present reviews will have to be evaluated by approaches in more complex environments. This will certainly involve an increasing number of gene knockouts involving the use of homologous recombination techniques and, probably, more refined methods relying on stage-, tissue-, and cell-lineage-specific removal of definable gene products. These approaches in conjunction with retroviral-mediated misexpression and more classical experiments involving in situ perturbation with specific antibodies should lead progressively to an understanding of the forces and factors that underlie the exquisite precision of wiring of the nervous system. To date, an impressive number of gene families has been uncovered, and the question remains open as to whether those families that have been identified are sufficient to account for axonal guidance and target finding in the developing nervous system. 